Original article

\title{
Early screening biomarker HbA1c and Hematocrit for gestational diabetes mellitus
}

\author{
Saswati Tripathy ${ }^{a, *}$, Anuradha Murugesan ${ }^{a}$, Kasthuri Natarajan ${ }^{\mathrm{b}}$, Balaji Ramraj ${ }^{\mathrm{c}}$, \\ Satyajit Mohapatra ${ }^{\mathrm{d}}$ \\ ${ }^{a}$ Department of Obstetrics and Gynaecology, India \\ ${ }^{\mathrm{b}}$ Department of Translational Medicine Research, India \\ ${ }^{\mathrm{c}}$ Department of Community Medicine, India \\ d Department of Pharmacology, SRM Medical College Hospital and Research Centre, SRM Institute of Science and Technology, SRM Nagar, Kattankulathur, 603203, \\ Kanchipuram, Chennai, TN, India
}

\section{A R T I C L E I N F O}

\section{Keywords:}

Biomarker

Glycated hemoglobin

Gestational diabetes mellitus

Hematocrit

\begin{abstract}
A B S T R A C T
Gestational diabetes mellitus (GDM) is a metabolic disturbance in pregnancy, traditionally defined as glucose intolerance with onset or first recognition during pregnancy with the outcome of perinatal and offspring complications. The common diagnostic criteria of oral glucose tolerance test in the second or third trimester of pregnant women help to detect the later complications of GDM. Early detection is lacking and highly essential to prevent GDM. We aim to assess the role of glycated hemoglobin and hematocrit for the early screening of GDM.

We recruited 403 study participants, collected demographic profiles and medical histories. The levels of glucose, hemoglobin ( $\mathrm{Hb})$, glycated hemoglobin (HbA1c), hematocrit (PCV), and thyroid-stimulating hormone (TSH) were measured in the serum samples of study participants. The ROC curve was plotted, cut-off, an area under the curve, specificity, and sensitivity of glycated hemoglobin and hematocrit was calculated using statistical software.

In our study, we observed there is a significant difference in the levels of $\mathrm{Hb}, \mathrm{PCV}$, and HbA1c between nonGDM and GDM patients. We found the prevalence of GDM $20.88 \%$ in our study population. An HbA1c cut of at $5.25 \%$ and PCV $34.5 \%$, the sensitivity and specificity of HbA1c as $64.4 \%$ and $36.1 \%$, the sensitivity and specificity of PCV as $58.6 \%$ and $43.1 \%$ respectively. The combined HbA1c \& PCV screening results had shown the sensitivity of $36.8 \%$ and specificity of $85.4 \%$ using the ROC curve.

In conclusion, the cut-off values, specificity, and sensitivity of HbA1c and Haematocrit and its combined calculation in the first trimester of pregnant women will be more specific and sensitive early screening tools for GDM.
\end{abstract}

\section{Introduction}

Gestational Diabetes Mellitus (GDM) is a complication in pregnancy associated with adverse outcomes in the maternal and fetal state with the increased risk of type 2 diabetes, macrosomia, obesity, cardiovascular diseases, morbidity, and mortality of fetus. The outcomes of perinatal and long-term offspring have a strong association with glycemic control in pregnancy. Some of the birth complications like birth injury, cesarean mode of delivery and large size fetus, fetal hypoxia, fetal hyperinsulinemia, hypoglycemia, polycythemia were well recognized high-risk complications in offsprings of GDM women. The long- term childhood complications include excess adiposity, neurodevelopmental impairment, glucose intolerance, disorders of glucose metabolism, type 2 diabetes mellitus, obesity, memory deficiency, psychological defects, developmental effects, and increased risk of autism has been documented in offsprings of GDM mothers. ${ }^{1}$

The criteria for screening tests for gestational diabetes mellitus were first suggested by Carpenter Coustan in $1982 .{ }^{2}$ National Diabetes Data Group (NDDG) in 1979 well explained the classification and diagnosis of diabetes mellitus and other categories of glucose intolerance. ${ }^{3}$ The diagnostic criteria were recommended by the international association of diabetes and pregnancy study groups (IADPSG) and adopted by the

\footnotetext{
* Corresponding author.

E-mail address: drsaswati78@gmail.com (S. Tripathy).
} 
world health organization (WHO). IADPSG criteria-based hyperglycemia and adverse pregnancy outcome (HAPO) studies found that the prevalence of GDM in the south Indian population is $17.8 \%{ }^{4-7}$ The standard value for fasting plasma glucose $<140 \mathrm{mg} / \mathrm{dL}$ and oral glucose tolerance test values $140-199 \mathrm{mg} / \mathrm{dL}$ was recommended by DIPSI criteria for gestational diabetes. $^{8}$

Current treatments for GDM include dietary lifestyle interventions, ${ }^{9,10}$ medical nutrition therapy with meal planning, ${ }^{11}$ moderate physical activities for $30 \mathrm{~min}$ per day, and first-line pharmacological therapy with insulin ${ }^{12,13}$ or oral agents like glyburide ${ }^{14}$ or metformin. ${ }^{15}$ Frequent monitoring of glucose levels, scheduled dietary and medications, physical exercise will be highly helpful to eradicate the complications of gestational diabetes mellitus.

The oral glucose tolerance test (OGTT) is the common diagnostic screening method for pregnant women performed at 24-28 weeks of gestation who may have risk factors of GDM. As OGTT is quite a timeconsuming, costly, low reproducibility, inconvenient confirmation of GDM, an alternative early screening strategy should be identified for the diagnosis and prevention of GDM risk in pregnant women. ${ }^{16}$

The glycated hemoglobin (HbA1c) is an irreversible non-enzymatic plasma protein glucose binding product, especially in hemoglobin. The mean level of plasma glucose on the erythrocyte is correlated with glycosylation degree. Measurement of this HbA1c will serve as an indicator of glucose control in 3-4 weeks of gestation may be used for early detection of women at risk of GDM. Very few prior studies examined and suggested HbA1c levels measured in the first trimester will be helpful for the prediction of GDM. ${ }^{16-19}$

Studies had shown that hematocrit (HCT or PCV) is associated with insulin sensitivity. There is a strong relationship between insulin resistance and high values of hematocrit in pregnancy. The high levels of hematocrit measured in the first trimester in perinatal care were found to be an independent predictor for GDM. ${ }^{20,21}$ Based on this background our primary aim is to examine the differences in levels of HbA1c and hematocrit between non- GDM and GDM, potential identification of cutoff values, specificity and sensitivity of HbA1c and PCV for GDM screening in the first trimester as well as to evaluate the association of HbA1c and hematocrit PCV in early prediction of GDM.

\section{Materials and methods}

\subsection{Study design}

Our study was designed as a cross-sectional study. The Institutional Ethics Committee approved this study. Study participants attending the department of obstetrics and gynecology, SRM Medical College Hospital and Research Centre, Tamilnadu, India were included after getting informed consent from the participating women.

\subsection{Participants criteria}

Pregnant women with and without gestational diabetes mellitus, between the age group of 18-35 years and, singleton pregnancy between 8 and 28 weeks of gestation were included in the study. Patients above 35 years, multiple pregnancies, with a history of type 2 diabetes, hypertension, hyperemesis, hematological disorders, organ dysfunction, thyroid disease, and in-vitro fertilization-embryo transfer were excluded from the study. GDM was diagnosed using International Association of Diabetes and Pregnancy Study Groups criteria (IADPSG): one or more of fasting plasma glucose level of $\geq 92 \mathrm{mg} / \mathrm{dL}$, 1-h plasma glucose level of $\geq 180 \mathrm{mg} / \mathrm{dL}$, and 2-h plasma glucose level of $\geq 153 \mathrm{mg} / \mathrm{dL}$ following a 75-g oral glucose tolerance test. Pregnant women with normal glucose tolerance and no other comorbidities are taken as control subjects.

\subsection{Demographic profile}

Study participants' profiles including age, height, weight, singleton or multiple pregnancies, period of gestation, family history and history of diabetes, other complications, were collected after receiving consent. Body mass index (BMI) was calculated based on the height and weight of the participants.

\subsection{Laboratory investigations}

Blood samples were collected from the study participants in a sterile vacutainer. $\mathrm{Hb}$, hematocrit PCV (packed cell volume), HbA1C was assessed in the blood samples. Serum samples were separated by centrifugation of blood at $3000 \mathrm{rpm}$ for $10 \mathrm{~min}$ and utilized for analysis of biochemical parameters using an automated clinical chemistry autoanalyzer (Beckman Coulter, California, USA) and hormones are analyzed in VITROS ${ }^{\circledR}$ ECiQ Immunodiagnostic System.

\subsection{Statistical analysis}

The statistical analysis was done using SPSS software version 21 . Students-test was done for the comparison of the parameters between non-GDM and GDM. A receiver operating characteristic (ROC) curve was plotted, sensitivity, specificity, and area under the curve was calculated to identify the cut-off values of HbA1c and hematocrit PCV. Pvalue $<0.05$ was considered to be statistically significant.

\section{Results and discussion}

\subsection{Study population}

Based on the inclusion and exclusion criteria of the participants, 403 subjects were included in the study. Among 403 subjects, 318 were nonGDM participants and 85 were GDM participants. The average age group of non-GDM subjects is $26.44 \pm 2.34$ years and GDM subjects are $27.0 \pm$ 2.87 years. The mean \pm SD values of BMI of non-GDM subjects were $26.54 \pm 3.45 \mathrm{~kg} / \mathrm{m}^{2}$ and GDM subjects were $30.10 \pm 2.33 \mathrm{~kg} / \mathrm{m}^{2}$ (Table 1).

\subsection{Clinical parameters}

Hemoglobin values of non-GDM subjects were $11.09 \pm 0.07 \mathrm{~g} / \mathrm{dL}$ and GDM subjects were $11.47 \pm 0.14 \mathrm{~g} / \mathrm{dL}$ Student t-test revealed a statistically significant difference in Hb values between GDM and nonGDM subjects $(P=0.020)$. The hematocrit PCV percent of non-GDM was $33.85 \pm 0.28 \%$ and GDM $34.85 \pm 0.37 \%$ which was statistically significant $(\mathrm{P}=0.034)$. HbA1c values for non-GDM were $5.18 \pm 0.04$ and GDM were $5.62 \pm 0.09$ with the p-value of 0.0001 . The thyroid levels of non-GDM and GDM was found to be $1.97 \pm 0.08 \mathrm{mU} / \mathrm{L}$ and $1.86 \pm 0.13 \mathrm{mU} / \mathrm{L}$ respectively (Table 1 ). We did not find any statistically significant difference in age, BMI, and thyroid levels between nonGDM and GDM participants in the study.

Table 1

Comparison of clinical characters of females with and without GDM.

\begin{tabular}{|c|c|c|c|c|}
\hline Parameters & $\begin{array}{l}\text { Non-GDM }(\mathrm{n}= \\
318)\end{array}$ & $\begin{array}{l}\text { GDM }(\mathrm{n}= \\
85)\end{array}$ & $\mathrm{T}$ test & $P$ value \\
\hline Age (years) & $26.44 \pm 2.34$ & $27.0 \pm 2.87$ & 0.567 & 0.568 \\
\hline $\begin{array}{l}\text { Body Mass Index (kg/ } \\
\mathrm{m}^{2} \text { ) }\end{array}$ & $26.54 \pm 3.45$ & $\begin{array}{l}30.10 \pm \\
2.33\end{array}$ & 0.477 & 0.634 \\
\hline Hemoglobin (gm/dl) & $11.09 \pm 0.07$ & $\begin{array}{l}11.47 \pm \\
0.14\end{array}$ & -2.345 & $0.020 *$ \\
\hline Hematocrit (\%) PCV & $33.85 \pm 0.28$ & $\begin{array}{l}34.85 \pm \\
0.37\end{array}$ & -1.2135 & $0.034^{*}$ \\
\hline HbA1c(\%) & $5.18 \pm 0.04$ & $5.62 \pm 0.09$ & -4.849 & $0.0001 *$ \\
\hline TSH (mU/L) & $1.97 \pm 0.08$ & $1.86 \pm 0.13$ & 0.708 & 0.479 \\
\hline
\end{tabular}

${ }^{*} \mathrm{P}<0.05$ is statistically significant. $\mathrm{P}$ value is calculated by student $t$-test. TSHThyroid stimulating hormone, PCV-packed cell volume, HbA1c-Glycated hemoglobin. 


\subsection{Cut-off value, specificity, and sensitivity of HbA1c and PCV in GDM}

A ROC curve was plotted to find the specificity and sensitivity of HbA1c at 12-28 weeks of gestation in GDM detection. In our study, the cut-off value of HbA1c was found to be $5.25 \%$ and PCV to be $34.5 \%$ (Fig. 1). The area under the ROC curve in HbA1c and PCV was found to be 0.715 and 0.604 respectively. HbA1c showed a sensitivity of $64.4 \%$ and specificity of $36.1 \%$. whereas PCV showed $58.6 \%$ of sensitivity and $43.1 \%$ specificity (Table 2). Combined HbA1c and PCV showed $36.8 \%$ of sensitivity and $85.4 \%$ of specificity under the ROC plot determination.

\section{Discussion}

Women with GDM have a greater risk of obstetrical hitches and adversative pregnancy consequences like preeclampsia and macrosomia. Previous studies had shown the worldwide prevalence of GDM as $4.8 \%$ and $15 \%$ by CDC and prevention whereas IADPSG (2010) and Seshiah et al. found $17.8 \%$ GDM prevalence in the south Indian population. ${ }^{4}$ The pooled GDM prevalence in Asia was reported as $11.5 \%$ Lee KW et al. $2018 .{ }^{22}$ In our study, we observed $20.88 \%$ of prevalence of GDM.

Over the previous years, HbA1c, a marker representative of the normal plasma glucose level in the preceding 8-12 weeks, has been recommended as a diagnostic indicator for diabetes mellitus in nonpregnant women. Compared to OGTT, HbA1c has advantages as the blood sample can be drawn in a non-fasting state and there is no need for ingestion of glucose or blood sampling at different time intervals. ${ }^{23,24}$

In nonpregnant women, though a level of $\geq 6.5 \%$ is suggested for diagnosis of diabetes, no harmony has been reached on the HbA1c efficacy for the diagnosis of diabetes during pregnancy. Still, HbA1c levels $\geq 6.5 \%$ have been used in detecting DM in early pregnancy on the basis that HbA1c levels in early pregnancy are comparable to those women who are not nonpregnant $(4.5 \%-5.7 \%$ in early pregnancy vs. $4.7 \%-$ $6.3 \%$ in non-pregnant women). ${ }^{25-27}$

Many investigators have tried to use $\mathrm{HbA1c}$ in the management of GDM patients. A study of Indian pregnant women conducted by Balaji and colleagues on HbA1c reported higher mean HbA1c values in GDM women compared to normal pregnant women $(5.96 \pm 0.63 \%$ [ $42 \mathrm{mmol} /$ $\mathrm{mol}]$ vs. $5.36 \pm 0.36 \%[35 \mathrm{mmol} / \mathrm{mol}]) .{ }^{28}$ One more study by Rajput et al. reported significantly higher HbA1c levels in GDM women $(5.73 \pm$ $0.34 \%$ [39 $\mathrm{mmol} / \mathrm{mol}]$ vs. $5.34 \pm 0.35 \%$ [35 mmol $/ \mathrm{mol}], \mathrm{p}<0.001$ ), and reported the analytical performance of $\mathrm{HbA1c}$ for GDM during the pregnant women in the second trimester. ${ }^{29}$

We also assessed the indicative performance of HbA1c for GDM compared to the reference method, 75-g OGTT. In this study, when an HbA1c level of $5.25 \%$ was used as a cut-off value, it showed high sensitivity (64.4\%), suggesting that measurement of HbA1c can be used as a screening tool for GDM, although the specificity was low (36.1.0\%).

In addition, there were variances in the cut-off value of HbA1c between our study and previous studies. We expect that these inconsistencies are caused by different ethnicities, and the mechanism of ethnic difference of HbA1c has yet been known clearly. Uneven relation between blood glucose levels and HbA1c can be explained by the hemoglobin glycation difference. ${ }^{30,31}$

Although HbA1c is not very virtuous at diagnosing GDM, it may have the potential to envisage adverse outcomes in pregnancy. HbA1c at pregnancy of 18-22weeks, or 32-36 weeks or HbA1c along with other clinical data in large sample size may help to screen for GDM and potentially reduce the number of OGTTs. Further, HbA1c could predict preeclampsia and birth weight.

\section{Conclusion}

HbA1c presented high sensitivity with comparatively low specificity for the GDM diagnosis in pregnant women and is a possible prognosticator of postpartum diabetes mellitus. HbA1c may have a potential for

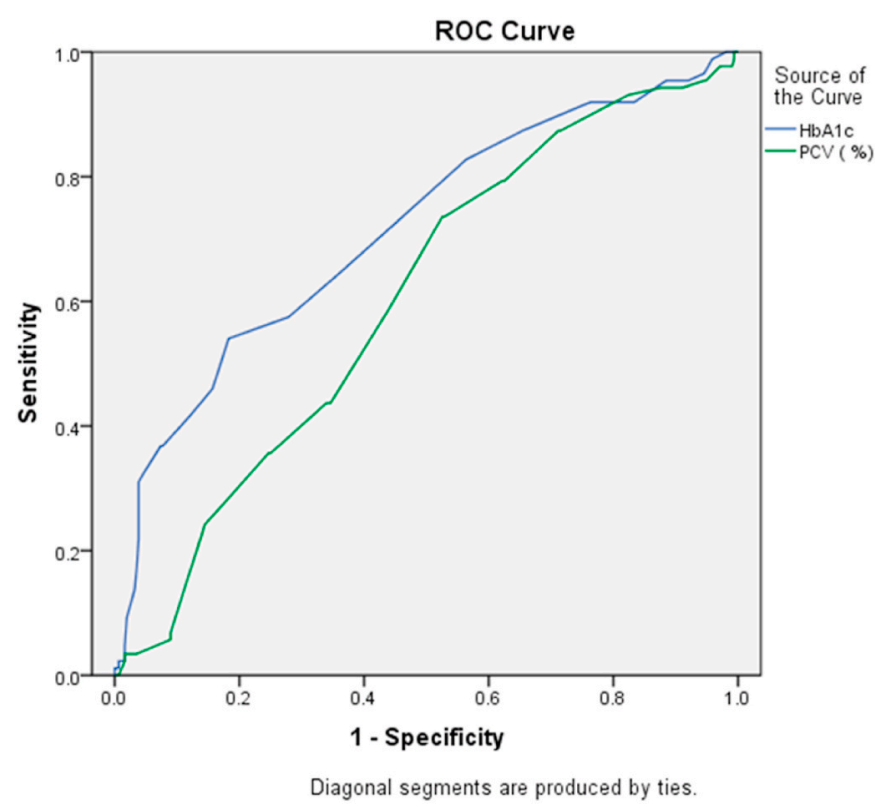

Fig. 1. ROC curve showing the sensitivity and specificity of HbA1c and PCV.

Table 2

Sensitivity and specificity of HbA1c and PCV from ROC curve.

\begin{tabular}{lllll}
\hline Parameters & Area under the curve & Cut-off value & Sensitivity & Specificity \\
\hline HbA1c & 0.715 & 5.25 & $64.4 \%$ & $36.1 \%$ \\
PCV & 0.604 & 34.5 & $58.6 \%$ & $43.1 \%$ \\
\hline
\end{tabular}

screening for GDM since it is likely to eradicate GDM in a considerable percentage of women and a less invasive substitute screening test for OGTT in GDM patients. Whether HbA1c alone or combined with other data like hematocrit can be useful in envisaging adverse outcomes in pregnancy among healthy women is unclear, and more research is needed as early management improves outcomes and decreases the forthcoming risk of diseases like DM, obesity, cardiovascular disease, and metabolic syndrome.

\section{Funding}

None.

\section{Declaration of competing interest}

None.

\section{References}

1 Szmuilowicz Emily D, MD MS, Josefson Jami L, MD MS, Boy E, Metzger MD. Gestational diabetes mellitus. Endocrinol Metab Clin N Am. 2019 September;48(3): 479-493.

2 Carpenter MW, Coustan DR. Criteria for screening tests for gestational diabetes. Am J Obstet Gynecol. 1982;144(7):768-773.

3 National Diabetes Data Group. Classification and diagnosis of diabetes mellitus and other categories of glucose intolerance. Diabetes. 1979;28:1039-1057.

4 Seshiah V, Balaji V, Balaji MS, et al. Prevalence of gestational diabetes mellitus in South India (Tamil Nadu)-a community based study. J Assoc Phys India. 2008 May; 56:329-333.

5 International Association of Diabetes Pregnancy Study Groups Consensus Panel, Metzger BE, Gabbe SG, Persson B, et al. International Association of Diabetes and pregnancy study groups recommendations on the diagnosis and classification of hyperglycemia in pregnancy. Diabetes Care. 2010;33(3):676-682.

6 HAPO Study Cooperative Research Group. Hyperglycemia and adverse pregnancy outcome (HAPO) study: associations with neonatal anthropometrics. Diabetes. 2009; 58(2):453-459.

7 Sacks DA, Hadden DR, Maresh M, et al. Frequency of gestational diabetes mellitus at collaborating centers based on IADPSG consensus panel-recommended criteria: the 
Hyperglycemia and Adverse Pregnancy Outcome (HAPO) Study. Diabetes Care. 2012; 35(3):526-528.

8 World Health Organization guideline. Diagnostic criteria and classification of hyperglycaemia first detected in pregnancy: a World Health Organization guideline. Diabetes Res Clin Pract. 2014;103(3):341-363.

9 Brown J, Alwan NA, West J, et al. Lifestyle interventions for the treatment of women with gestational diabetes. Cochrane Database Syst Rev. 2017;5:CD011970.

10 Viana LV, Gross JL, Azevedo MJ. Dietary intervention in patients with gestational diabetes mellitus: a systematic review and meta-analysis of randomized clinical trials on maternal and newborn outcomes. Diabetes Care. 2014;37(12):3345-3355.

11 Crowther CA, Hiller JE, Moss JR, et al. Effect of treatment of gestational diabetes mellitus on pregnancy outcomes. N Engl J Med. 2005;352(24):2477-2486.

12 Committee on Practice Bulletins-Obstetrics. ACOG Practice Bulletin No. 190: gestational diabetes mellitus. Obstet Gynecol. 2018;131(2):e49-64.

13 American Diabetes Association. Standards of medical care in diabetes - 2019. Diabetes Care. 2019;42(Supplement 1):S1-193.

14 Langer O, Conway DL, Berkus MD, et al. A comparison of glyburide and insulin in women with gestational diabetes mellitus. N Engl J Med. 2000;343(16):1134-1138.

15 Balsells M, Garcia-Patterson A, Sola I, et al. Glibenclamide, metformin, and insulin for the treatment of gestational diabetes: a systematic review and meta-analysis. BMJ. 2015;350:h102.

16 Amylidi S, et al. First-trimester glycosylated hemoglobin in women at high risk for gestational diabetes. Acta Obstet Gynecol Scand. 2016;95:93-97.

17 Benaiges D, et al. Is frst-trimester HbA1c useful in the diagnosis of gestational diabetes? Diabetes Res Clin Pract. 2017;133:85-91.

18 Ho Y-R, et al. Associations of mid-pregnancy HbA1c with gestational diabetes and risk of adverse pregnancy outcomes in high-risk Taiwanese women. PLoS One. 2017; 12, e0177563.

19 Hinkle Stefanie N, Tsai MichaelY, Rawal1 Shristi, Albert Paul S, Zhang Cuilin. HbA1c measured in the first trimester of pregnancy and the association with gestational diabetes. Sci Rep. 2018;8:12249.
20 Hanley AJ, Retnakaran R, Qi Y, et al. Association of hematological parameters with insulin resistance and beta-cell dysfunction in nondiabetic subjects. J Clin Endocrinol Metab. 2009;94:3824-3832.

21 Tan PC, Chai JN, Ling LP, Omar SZ. Maternal hemoglobin level and red cell indices as predictors of gestational diabetes in a multi-ethnic Asian population. Clin Exp Obstet Gynecol. 2011;38:150-154.

22 Lee KW, Ching SM, Ramachandran V, et al. Prevalence and risk factors of gestational diabetes mellitus in Asia: a systematic review and meta-analysis. BMC Pregnancy Childbirth. 2018;18:494. https://doi.org/10.1186/s12884-018-2131-4.

23 Nathan DM, Turgeon H, Regan S. Relationship between glycated hemoglobin levels and mean glucose levels over time. Diabetologia. 2007;50:2239-2244.

24 International Expert Committee. International Expert Committee report on the role of the A1C assay in the diagnosis of diabetes. Diabetes Care. 2009;32:1327-1334.

25 Radder JK, van Roosmalen J. HbA1c in healthy, pregnant women. Neth J Med. 2005; 63:256-259.

26 Nielsen LR, Ekbom P, Damm P, et al. HbA1c levels are significantly lower in early and late pregnancy. Diabetes Care. 2004;27:1200-1201.

27 Battarbee AN, Grant JH, Vladutiu CJ, et al. Hemoglobin A1c and early gestational diabetes. $J$ Wom Health. 2020;29(12):1559-1563. https://doi.org/10.1089/ jwh.2019.8203.

28 Balaji V, Madhuri BS, Ashalatha S, Sheela S, Suresh S, Seshiah V. HbA1C in gestational diabetes mellitus in Asian Indian women. Diabetes Care. 2007;30: 1865-1867.

29 Rajput R, Yogesh Y, Rajput M, Nanda S. Utility of HbA1c for diagnosis of gestational diabetes mellitus. Diabetes Res Clin Pract. 2012;98:104-107.

30 Wolffenbuttel BH, Herman WH, Gross JL, Dharmalingam M, Jiang HH, Hardin DS. Ethnic differences in glycemic markers in patients with type 2 diabetes. Diabetes Care. 2013;36:2931-2936.

31 Herman WH, Dungan KM, Wolffenbuttel BH, et al. Racial and ethnic differences in mean plasma glucose, hemoglobin A1c, and 1,5-anhydroglucitol in over 2000 patients with type 2 diabetes. J Clin Endocrinol Metab. 2009;94:1689-1694. 